\title{
MORE ABOUT JENSEN'S INEQUALITY AND CAUCHY'S MEANS FOR SUPERQUADRATIC FUNCTIONS
}

\author{
S. Abramovich, G. FARID AND J. PEČArić
}

Abstract. One of the many inequalities that superquadratic functions satisfy is the parallelogram inequality

$$
f(u+v)+f(u-v) \geqslant 2 f(u)+2 f(v) .
$$

In this paper, we present Cauchy means for superquadratic functions and other mean value theorems. We show also positive semi-definiteness, log-convexity, exponential convexity of certain set of functions.

Mathematics subject classification (2010): Primary 26A51; Secondary 26A46, 26 A48.

Keywords and phrases: Jensen's inequality, log-convex function, linear functional, superquadracity, mean value theorems.

\section{REFERENCES}

[1] S. Abramovich, G. Jameson, G. Sinnamon, Inequalities for averages of convex and superquadratic functions, JIPAM. J. Inequal. Pure Appl. Math. 5, 4 (2004), Article 91, 14 pp.

[2] S. Abramovich, G. Jameson, G. Sinnamon, Refining Jensen's inequality, Bull. Math. Soc. Sci. Math. Roumanie 47(95), 1-2 (2004), 3-14.

[3] M. Anwar, J. Jakšetić, J. PeČarić, AtiQ UR Rehman, Exponential convexity, positive semidefinite matrices and fundamental inequalities, J. Math. Inequal. 4, 2 (2010), 171-189.

[4] M. Anwar, Naveed Latif, J. PeČarić, Cauchy means of the Popoviciu type, J. Inequal. Appl. 2009, Art. ID 628051, 16 pp.

[5] M. Anwar, J. PeČArić, Cauchy means of Levinson type, JIPAM. J. Inequal. Pure Appl. Math. 9, 4 (2008), Article 120, 8 pp.

[6] M. Anwar, J. PeČARIĆ, New means of Cauchy's type, J. Inequal. Appl. 2008, Art. ID 163202, 10 pp.

[7] S. BAnić, Superquadratic functions, thesis, University of Zagreb.

[8] S. BAnIĆ, S. VARošAneC, Functional inequalities for superquadratic functions, Int. J. Pure Appl. Math. 43, 4 (2008), 537-549.

[9] I. I. HiRsChman AND D. V. WIdDeR, The convolution transform, Princeton University Press, Princeton, N. J., 1955.

[10] D. S. Mitrinović, J. PeČArić, A. M. Fink, Classical and new inequalities in analysis, Kluwer Academic Publishers Group, Dordrecht, 1993.

[11] J. PeČArić, F. Proschan, Y. L. Tong., Convex functions, partial orderings, and statistical applications, Academic Press, Inc., Boston, MA, 1992.

[12] J. PeČAriĆ, I. Perić, H. M. SRivastaVA, A family of the Cauchy type mean-value theorems, J. Math. Anal. Appl. 306, 2 (2005), 730-739.

[13] W. SiER PIŃS KI, Sur les fonctions convexes mesurables, Fund. Math. 1 (1920), 125-128. 